\title{
A New TURBO LMS Beamforming for Mobile Communication
}

\author{
H.V Kumaraswamy \\ Asst. Professor \\ Dept of Telecommunication, \\ R.V.C.E
}

\author{
Aaquib Nawaz.S \\ Dept of Telecommunication \\ R.V.C.E
}

\author{
Syed Younus \\ Dept of Telecommunication \\ R.V.C.E
}

\begin{abstract}
Array processing involves manipulation of signals induced on various antenna elements. Its capabilities of steering nulls to reduce co-channel interferences and pointing independent beams towards various users, as well as its ability to provide estimate of directions of radiating sources, make it attractive to the mobile communications system designer.

In this paper existing Least Mean Square (LMS) algorithm is modified to obtain lesser Mean Square Error (MSE) by using leaky factor in the weight updation.A novel approach named as TURBO LMS is proposed which reduces the Mean Square Error and increases the convergence speed by a large amount as compared to existing beamforming algorithms. Simulation of Beamforming algorithms namely Least Mean Square (LMS), Leaky Least Mean Square (LLMS) and novel TURBO LMS algorithm are done for various look directions and jammer configurations and their MSE characteristics are compared. MATLAB simulation shows that the proposed TURBO LMS algorithm has improved convergence as compared to existing beamforming algorithms. Performance of TURBO LMS algorithm is measured by varying number of array elements.
\end{abstract}

\section{Keywords}

Adaptive array beamforming, LMS algorithm, TURBO LMS and Mean Square Error.

\section{INTRODUCTION}

In the recent years, adaptive or smart antennas have become a key component for various wireless applications, such as radar, sonar, and $4 \mathrm{G}$ cellular mobile communications [1]. Its use leads to an increase in the detection range of radar and sonar systems and it also increases the capacity of mobile Communication systems. Adaptive filtering [2] has been, and still is, an area of active research that plays an active role in an ever increasing number of applications such as noise cancellation, channel estimation, channel equalization and acoustic echo cancellation. The Least Mean Square (LMS) and its normalized version (NLMS) are the workhorses of adaptive filtering. In the presence of correlated input signals, the LMS and NLMS algorithms have extremely slow convergence rates. To solve this problem a number of adaptive filtering structures based on affine subspace projections [14], data reusing adaptive algorithms, block adaptive filters and multirate techniques have been proposed [4]. In all these algorithms the step-size can change the convergence speed and the steady-state mean square error. Variable step size algorithms for beamforming are developed based on certain modifications to Finite-Impulse Response Wiener filtering [10]. Griffths and Variable Step Size Griffths (VSSG) algorithms have less misadjustment and better convergence as compared to VSS-LMS[11].

TURBO LMS algorithm described in this paper provides excellent convergence characteristics by having four Beamforming blocks executing in parallel. It uses two conventional LMS blocks, Convergence Speediness Modules (CSM) block and Speediness Module(SM) block. Small Modifications to existing Variable Step Size algorithm are made to obtain EDNSS and ENSS, which have reduction in computation time and complexity as compared to existing Variable Step Size algorithms.

\section{BASIC \\ PRINCIPLES \\ OF BEAMFORMING}

A beamformer is a set of sensors (antennas), arranged in a linear fashion (Uniform Linear Array), that extract spatial information from the waves emitted by signal sources in order to steer the beam electronically towards the look direction and nulls in the jammer directions. The signal received at sensor is sent for computation of weights.

Let $x(n)$ be the complex vector of $\mathrm{L}$ elements of array observations. $w(n)$ is the complex weight vector, $\mathrm{L}$ is the number of array sensors and $s(n)$ is sequence of training signal samples.

The output of a linear beamformer is given by

$y(n)=w(n)^{T} x(n)$

Where, $y(n)$ is the output of beamformer, Coefficients $w(n)$ are usually estimated through the minimization of error $e(n)$.

$e(n)$ is given by

$e(n)=s(n)-y(n)$

\section{LEAST MEAN SQUARE ALGORITHM (LMS)}

The LMS algorithm is the most widely used adaptive beamforming algorithm, being employed in several communication applications. The LMS algorithm changes the weight vector $w(n)$ along the direction of the estimated gradient based on the steepest descent method. The weight vector updation for LMS algorithm is given by

$w(n+1)=w(n)+\mu e(n) x^{*}(n)$ 


\section{LEAKY LMS (LLMS) BEAMFORMER}

In this algorithm noise is added to the autocorrelation matrix by using Leaky factor. The autocorrelation matrix $R_{x x}$ used in LMS algorithm in some cases has zero Eigen values. This causes LMS algorithm to have un-damped and un-driven modes. Since it is possible for these un-damped modes to become unstable. It is important to stabilize the LMS by forcing these modes to zero. One way to accomplish this is to introduce leakage coefficient $\gamma$, which is in the range $0<\gamma<1$ into auto correlation matrix, step size calculation and weight vector equation. The weight update equation of LLMS is given by

$$
W(n+1)=[1-\mu \gamma] W(n)+\mu e^{*}(n) x(n) \mid
$$

Where, $\mu$ is step size computed by using

$$
\mu=\frac{2}{\lambda_{\max }+\gamma}
$$

Where $\lambda_{\max }$ is the maximum Eigen value obtained from Eigen value decomposition of correlation matrix with a leaky factor along diagonal elements added linearly.

\section{TURBO LMS ALGORITHM}

LMS Beamforming algorithm has low computation complexity however; its convergence rate is slow. In this section, a novel implementation scheme for LMS algorithm is presented to form a modified LMS algorithm. The scheme is similar to the structure of Turbo decoder, which consists of one feedback module called as converse-speediness module, two LMS algorithm modules and one speediness module, which connects two LMS algorithm modules.

\section{SCHEME OF TURBO LMS (T-LMS) ALGORITHM}

The proposed scheme is similar to the structure of the Turbo decoder, which is shown in Figure 1. It has four components: LMS algorithm ModuleI (LMSAMI), LMS algorithm ModuleII (LMSAM II), Speediness Module (SM) and ConverseSpeediness Module (CSM).

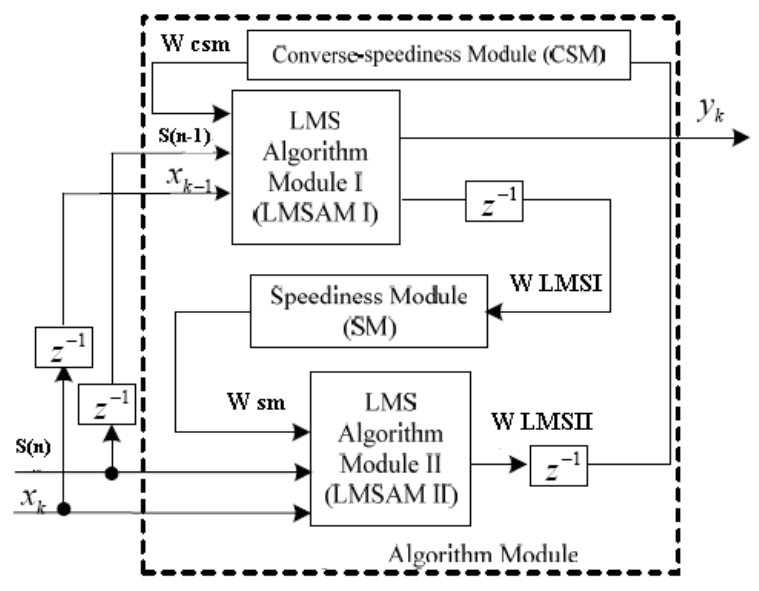

Figure 1: TURBO LMS Block Diagram
The method used in SM is Aitken method [3] which is used as quick iterative method for equation root finding. LMSAM I and LMSAM II both use conventional LMS algorithm. The main purpose of LMS algorithm is to find a proper weight vector $\left(\mathbf{w}_{\boldsymbol{k}}\right)$ which can make the MSE of the system output and the reference signal minimum. The weight vector $\left(\mathrm{w}_{\mathrm{k}}\right)$ would be stable for larger iterations i.e

$$
\lim _{k \rightarrow \infty} \frac{w_{k-1}-w_{s}}{w_{k}-w_{s}}=\text { const } \quad, k=1,2, \ldots
$$

Where, $k$ is the number of iterations, and $\mathbf{w}_{\mathbf{s}}$ is the stable-state weight vector. Since equation (6) satisfies the restrictive condition of Aitken method with good performance of quick convergence, we choose Aitken method for SM. As shown in Figure 1, we can see that SM can output a weight vector $\left(w_{s m}\right)$ which is much more approximate to the stable-state weight vector $\left(\mathrm{w}_{\mathrm{s}}\right)$ than the weight vector $\left(w_{L M S I}\right)$ of LMSAMI. CSM gets the weight vector $w_{c s m}$ from calculating the weight vector ( $w_{\text {LMS II }}$ ) of LMSAMII and then feeds back $w_{c s m}$ to LMSAMI. $w_{c s m}$ obtained in CSM is closer to the stable-state weight vector $\left(\mathbf{w}_{\mathbf{s}}\right)$ than the weight vector $\left(w_{L M S I}\right)$ computing in LMSAMI. Therefore, the convergence rates of both LMSAMI and LMSAM II are accelerating greatly.

\section{A CONVERGENCE SPEEDY MODULE} (CSM)

The array weights of Convergence Speedy Module is given by

$w_{c s m}(n+1)=w_{c s m}(n)+\left(\left[I-2 \mu_{c s m} R_{x}\right]^{-1}\left[v_{k}-2 \mu P_{k}\right]\right)$

Where, $w_{c s m}(n+1)$ are the Lx1 updated array weights, $I$ is LxL identity matrix, $R_{x}$ is the $\mathrm{LxL}$ autocorrelation matrix, $v_{k}$ are Lx1 array weights obtained from LMSII module initially this value is zero and $P_{k}$ is the cross-correlation between array observation vector and reference signal and $\mu$ is the step size given by

$\mu_{c s m}=\frac{1}{3 \operatorname{tr}\left(R_{x}\right)}$

Where $\operatorname{tr}\left(R_{x}\right)$ is the trace of autocorrelation matrix.

\section{B SPEEDINESS MODULE (SM)}

As per the Aitkin method [3] the array weights of SM module can be found out using

$$
\begin{aligned}
& w_{s m}=\psi\left(\psi\left(w_{k}\right)\right)-\frac{\text { power }\left\{\psi\left(\psi\left(w_{k}\right)\right)-\psi\left(w_{k}\right)\right\}}{\psi\left(\psi\left(w_{k}\right)\right)-2 \psi\left(w_{k}\right)+w_{k}} \\
& \psi\left(w_{k}\right)=M_{K} w_{K}+N_{K}
\end{aligned}
$$

Where, $M_{K}=I-2 \mu R_{K}$ and $N_{K}=2 \mu P_{k}$ 


\section{SIMULATION RESULTS}

The performance of Beamforming algorithms has been studied by means of MATLAB simulation. For comparison purposes, results obtained with the conventional LMS and VSS-LMS algorithm are also presented.

For Simulation the following assumptions are considered

1. Mutual Coupling effects are zero between antenna elements.

2. Distance between antenna elements is $\lambda / 2$ an optimum value to avoid grating lobes.

3. Look Direction and jammer directions have been determined apriori.

4. Number of array elements $=100$.

Case (i): Beamforming Result for LMS

Look Direction $=45^{0}$

Interference Directions $=10^{\circ}$ and $30^{\circ}$.

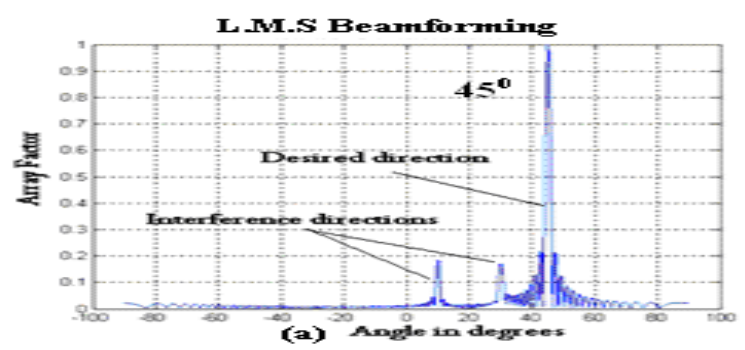

Figure (2): Beam Plot of LMS algorithm

From the Figure(2) it is clear that $L M S$ algorithm is able to form the main beam in the look direction of $45^{\circ}$ and small radiations in the interferer directions.

\section{Case (ii): Beamforming Results of LLMS}

$\mathrm{L}=$ Number of array elements $=100$.

$\theta_{o}=$ Look Direction $=20^{\circ}$

Jamming sources $=10^{\circ}, 30^{\circ}$ and $40^{\circ}$

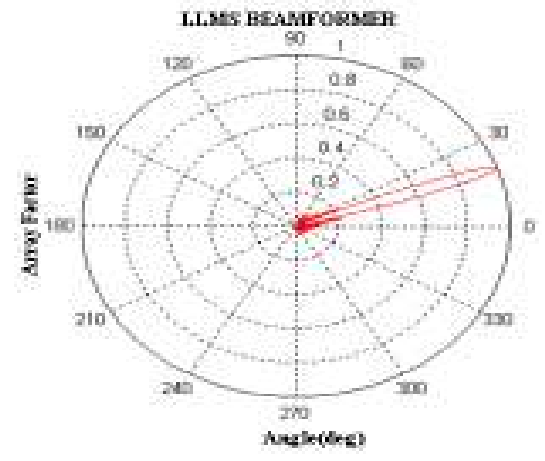

Figure (3):Beamforming using LLMS
Figure (3) shows beam formed at an angle $20^{\circ}$ by using LLMS Beamforming algorithm

\section{Case (iii): Beamforming Results for TURBO LMS}

Look Direction $=45^{0}$

Interference Directions $=10^{\circ}$ and $30^{\circ}$

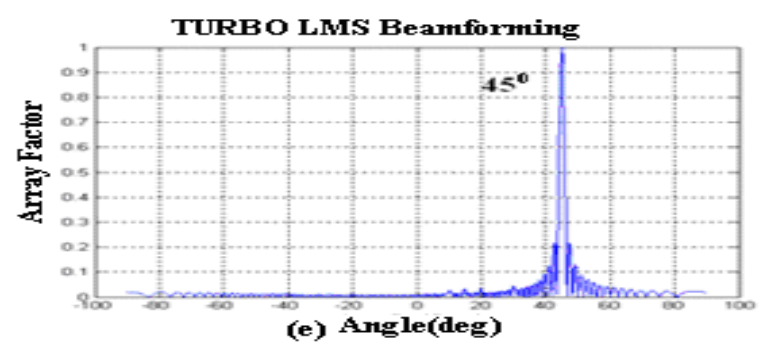

Figure (4): Beam Plot of TURBO- LMS algorithm

From the Figure(4) it is clear that TURBO-LMS algorithm is able to form the main beam in the look direction of $45^{\circ}$ and deep nulls in direction of interferers.

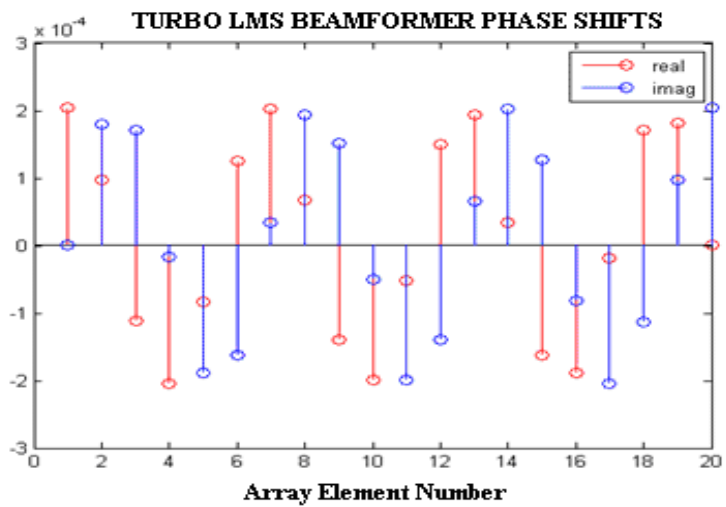

Figure (5): Complex Phase Shifts of TURBO-LMS

Figure (5) shows the complex phase shifts computed using TURBO LMS Beamforming algorithm which is applied to individual element.

Case (IV): MSE comparison curve of Beamforming algorithms

$\mathrm{L}=$ Number of Array elements $=100$.

Table1: Input to Beamforming algorithms

\begin{tabular}{|c|c|}
\hline Look Direction & Jammer Directions \\
\hline $45^{0}$ & {$\left[10^{0}, 20^{0}, 30^{\circ}, 60^{\circ}\right]$} \\
\hline
\end{tabular}




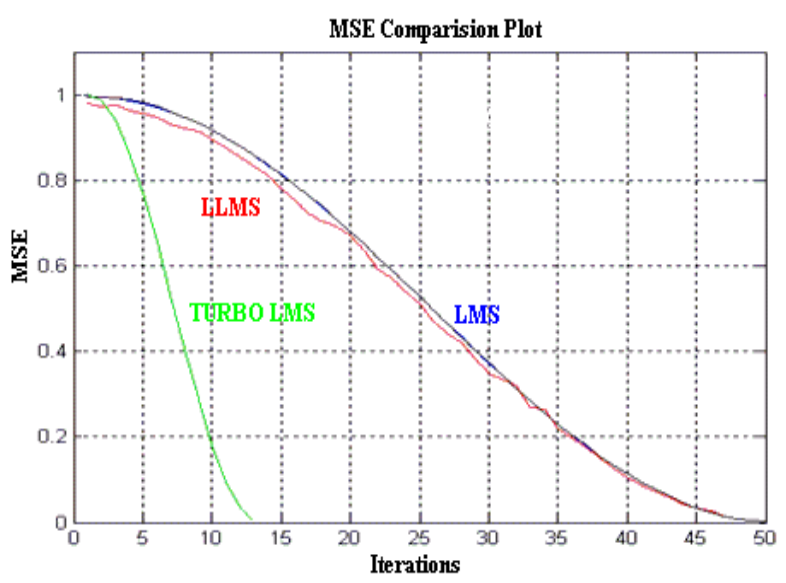

Figure (6): MSE comparisons Curve for Beamforming

From the simulation result of various beamforming algorithms as shown in Figure(6) LMS, LLMS converge for 48 iterations but TURBO LMS converges for 12 iterations. Hence TURBO LMS is the best suited as compared to existing Beamforming algorithms in terms MSE characteristics and convergence.

\section{Case (v): Tradeoff Between Beam width and RF cost}

The performance of TURBO LMS algorithm was simulated by varying number of array elements.

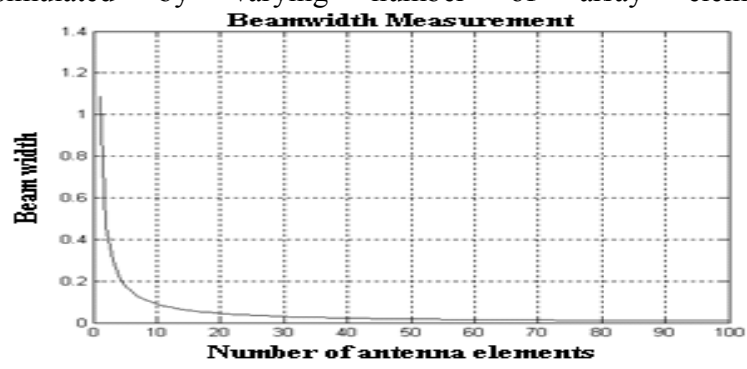

Figure (7): Measured Beam width by varying number of antenna elements

From the Simulation curve of Figure (6) we can conclude that there is a tradeoff between RF cost (number of antenna elements) and reduction in beam width (directivity).

\section{Case (vi): Execution Speed of Beamforming}

The performance of Beamforming algorithms with respect to execution speed is compared.

Table (2): Execution Time for Beamforming

\begin{tabular}{|c|c|}
\hline Beamforming algorithm & Execution Time in seconds \\
\hline L.M.S & 0.8541 \\
\hline LLMS & 0.4780 \\
\hline TURBO LMS & 0.4297 \\
\hline
\end{tabular}

From the Table (2) it is clear that TURBO LMS Beamforming algorithm takes least amount of execution time as compared to other algorithms. The execution speed is measured by using timer options in MATLAB. Execution speed is measured by taking average of 200 runs.

Case (vii): Error Vector Magnitude (EVM) measurement of Beamforming algorithms

For a complex signal, it is also convenient to make use of the Error Vector Magnitude (EVM) as an accurate measure of any distortion introduced by the adaptive scheme on the received signal [8].

The EVM is given by

$$
E V M_{r m s}=\sqrt{\frac{1}{K P_{0}} \sum_{j=1}^{K}\left|S_{r}(j)-S_{t}(j)\right|^{2}}
$$

Where, $K$ is the number of observations used, $S_{r}(j)$ is the normalized $j^{\text {th }}$ output of the beamformer and $S_{t}(j)$ is the $j^{\text {th }}$ transmit signal. $P_{0}$ is the normalized transmit signal power.

$\mathrm{K}=$ Number of observations $=100$.

Table (3): Simulation Results of EVM

\begin{tabular}{|c|c|}
\hline Algorithm & EVM \\
\hline LMS & 0.9870 \\
\hline LLMS & 0.9760 \\
\hline TURBO LMS & 0.9582 \\
\hline
\end{tabular}

From the MATLAB simulation results of Table(3) it is clear that the EVM of TURBO-LMS algorithm is less as compared to other algorithms.

\section{Case(viii): Effect of Change in Step Size on weight magnitude}

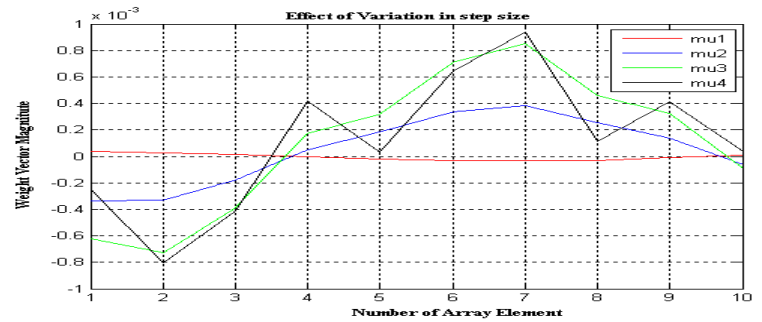

Figure(9): Step Size Variation Effects

The performance of TURBO LMS algorithm is studied by varying the step size.As the step size is incresed the weigth vector magnitude has large amount of variations. Hence it is good to choose smaller step size for better performance as shown in simulation curve of Figure(9).

\section{CONCLUSION}

A new adaptive algorithm for beamforming called TURBO LMS algorithm is presented and analyzed. The performance is compared with existing LMS algorithm and new LLMS algorithm. 
It is shown that the proposed TURBO LMS algorithm can achieve rapid convergence i.e LMS and LLMS converges for 48 iterations where as TURBO LMS converges for about 12 iterations. Simulation results also show a trade off between number of array elements and directivity. Unlike conventional LMS and LLMS, TURBO LMS takes less amount of execution time.

\section{REFERENCES}

[1] Martin-Sacristan, D., et al., "On the Way toward ForthGeneration Mobile: 3GPP LTE-Advanced", EURASIP Journal on Wireless Communications and Networking, vol. 2009, Jun. 2009.

[2] Stine, J.A., "Exploiting smart antennas in wireless mesh networks using contention access", IEEE Trans. On Wireless Communications, vol. 13(2), pp. 38-49, 2006.

[3] Qian Liu, Minglu Jin and Aifei Liu, "A Modified LMS Algorithm with Turbo-like Scheme", 3rd International Conference on Innovative Computing Information and Control, IEEE 2008, June 2008, pp-534.

[4] Godara, "Applications of Antenna Arrays to Mobile Communications, Part I: Performance Improvement, Feasibility and System Considerations", IEEE Proceedings, July 1997, Vol 85, pp 102-129.

[5] Guancheng Lin, Yaan Li, and Beili Jin, "Research on Support Vector Machines Framework for Uniform Arrays Beamforming", IEEE International Conference on Intelligent Computation Technology and Automation, 2010

[6] Ahmad Al-Diab, Constantinos Sourkounis, "Variable Step Size P\&O MPPT Algorithm for PV Systems", IEEE 12th International Conference on Optimization of Electrical and Electronic Equipment, pp 1097-1102.

[7] Zhao, S., Z. Man, and S. Khoo, "A Fast Variable Step-Size LMS Algorithm with System Identification", $2^{\text {nd }}$ IEEE Conference on Industrial Electronics and Applications, Harbin, China, pp. 2340-2345, May 2007.

[8] T.Lo, H.Leung, J.Litva, S.haykin, "Fractal characterisation of seascattered signals and detection of sea-surface targets", IEEE Proceedings, vol.140, pp. 243-250, August 1993.

[9] M.Martorella, F. Berizzi, E.D. Mese, "On the fractal dimension of sea surface backscattered signal at low grazing angle”, IEEE Trans. Antennas \& Propagation, vol. 52, pp. 1193-1204, May 2004.

[10] H.V Kumaraswamy, Aaquib Nawaz.S, Vidyasagar Ture, "Beamforming using VSS-LMS for Mobile Communication", 4th National Conference on Recent Trends in Communication, Electronics \& Information Technology, $23^{\text {rd }}$ and $24^{\text {th }}$ April 2010.pp- 102-105.

[11] Kumaraswamy H.V, S.Ravishankar, "Implementation of Variable Step Size Griffths algorithm for adaptive beamforming", Proccedings of IEEE international symposium on microwaves, 2008, Vol-8, pp: 31-35.

[12] F. Berizzi, E.Dalle Mese, M. Martorella, "A sea surface fractal model for ocean remote sensing", International Journal of Remote Sensing, vol.25, pp. 1265-1270, 2004.
[13] A. Collaro, G. Franceschetti, M. Migliaccio, D. Riccio, "Gaussian rough surfaces and Kirchhoff approxination", IEEE Trans. on Antennas \& Propagation, vol. 47, pp. 392398, 1999.

[14] H.-C. Shin, A. H. Sayed, and W.-J. Song, "Variable stepsize NLMS and affine projection algorithms," IEEE Signal Process. Lett., vol. 11, no. 2, pp. 132-135, Feb. 2004.

[15] Sungsoo, Seungwon and Tapan K sarkar, "An Adaptive Beamforming Algorithm with a Linear Complexity for a Multipath Fading CDMA Channel", IEICE Transaction Communication, Vol E84-B, No 8, August 2001, pp 23172320 .

[16] Okello, Y.Fukui, I.Nakanishi, Kobayashi, M "A New Modified Variable Step Size Algorithm for the LMS algorithm", IEEE Proceedings of the International Symposium on Circuits and Systems, June 1998 , Vol 5,pp $170-173$.

[17] K. S. Gilhousen, I. M. Jacobs, R. Padovani, A. J. Viterbi, J. Lindsay A. Weaver, and C. E. Wheatley . "On the capacity of a cellular CDMA system". IEEE Dansactions on Vehicular Technology, 40(2):303-312,1991.

[18] A. Jalali and P. Mermelstein. "Effects ofdiversity, power control, and bandwidth on the capacity of microcellular CDMA systems". IEEE Journal on Selected Areas in Communications, 12(5):952-96 1, 1994.

[19] R. H. Kwong and E. W. Johnston, "A variable step size LMS algorithm," IEEE Trans. Signal Process., vol. 40, no. 7, pp. 1633-1642, Jul. 1992.

[20] V. J. Mathews and Z. Xie, "A stochastic gradient adaptive filter with gradient adaptive step size," IEEE Trans. Signal Process., vol. 41, no. 6, pp. 2075-2087, June 1993.

\section{DATA OF AUTHORS}

H.V.Kumaraswamy, Asst Professor, Department of Telecommunication, RVCE, Bangalore. Currently pursuing Ph.D degree in Smart Antennas. Presented papers at several International IEEE conferences and authored Signals and Systems text book. Current areas of interest include Adaptive Beamforming, Dielectric Lens antenna, Digital Signal Processing, Signals \& Systems, Advanced Digital Communication, Statistical Signal Processing and Multi rate Filter Banks.

Aaquib Nawaz.S, Dept. of Telecommunication, RVCE, Bangalore. Is a faculty in the Dept of Telecommunication, RVCE. Areas of interest include Adaptive Filter Theory, Antenna Theory and Design, Broadband Access Networks, Wireless Communication and Networking.

Syed Younus, Dept. of Telecommunication, RVCE, Bangalore. Areas of interest are Adaptive Beamforming, Network Protocol Design, Multirate Filter Banks, Wireless communication and Antenna Theory \& Design. 